\title{
Effects of Dietary Green Rooibos on Physical Endurance in Swimming Mice
}

\author{
Nakamichi Watanabe $^{{ }^{*}}$, Yurie Hara ${ }^{2}$, Satomi Sakuda ${ }^{1}$, Yoriko Mori ${ }^{1}$, \\ Yutaka Furuya $^{3}$, Tsuyoshi Watanabe ${ }^{3}$ \\ ${ }^{1}$ Department of Human Science and Design, Showa Women's University, Tokyo, Japan; ${ }^{2}$ Department of Human Sciences and Design,
Graduate School of Showa Women’s University, Tokyo, Japan; ${ }^{3}$ Tama biochemical Co., Ltd., Tokyo, Japan. \\ Email: "nakamich@swu.ac.jp
}

Received October $4^{\text {th }}, 2013$; revised November $4^{\text {th }}$, 2013; accepted November $11^{\text {th }}, 2013$

Copyright (C) 2014 Nakamichi Watanabe et al. This is an open access article distributed under the Creative Commons Attribution License, which permits unrestricted use, distribution, and reproduction in any medium, provided the original work is properly cited. In accordance of the Creative Commons Attribution License all Copyrights (C) 2014 are reserved for SCIRP and the owner of the intellectual property Nakamichi Watanabe et al. All Copyright (c) 2014 are guarded by law and by SCIRP as a guardian.

\section{ABSTRACT}

Green rooibos made from unfermented rooibos leaves contains high amounts of aspalathin. Dietary green rooibos is expected to increase physical endurance and decrease physical fatigue, because aspalathin has antioxidant activity and stimulates glucose uptake in muscle tissue. The aim of this study was to validate the effects of green rooibos and aspalathin by using a swimming mouse model. Dietary green rooibos and aspalathin tended to increase swimming time in mice. This beneficial effect might be induced by increased glycogen accumulation in the liver and skeletal muscles.

\section{KEYWORDS}

\section{Aspalathin; Fatigue; Glycogen; Physical Endurance; Rooibos}

\section{Introduction}

Rooibos is a bushy leguminous tree cultivated in mountain ranges in South Africa. Rooibos tea, made by the fermentation of the leaves, has been utilized as a medical herbal product with sedative, digestive, and anti-inflammatory effects.

Recently, a new rooibos tea prepared from unfermented leaves called green rooibos was introduced. Green rooibos (Aspalathus linearis) has a high content of aspalathin, a C-glycosylflavonoid with a molecular weight of 452.5 and a peculiar molecular structure wherein glucose is linked to dihydrochalcone via a carbon-carbon bond. Aspalathin has been suggested to have antioxidant activity in vivo [1], and green rooibos has a substantially higher aspalathin content than that of fermented rooibos [2]. Given that free radicals are considered as one of the factors that terminate the endurance to muscle effort in mice [3], dietary green rooibos is expected to exert anti-fatigue effects.

Moreover, dietary aspalathin was suggested to stimu-

${ }^{*}$ Corresponding author. late glucose uptake in muscle tissue [4,5], which is related to increased translocation of glucose transporter type 4 (GLUT4) to the plasma membrane via activation of 5' AMP-activated protein kinase (AMPK) [6]. If aspalathin promotes the supply of blood glucose to skeletal muscles, physical endurance might be increased.

Therefore, the intake of green rooibos is expected to increase physical endurance and decrease physical fatigue. The aim of this study is to validate the effects of green rooibos and aspalathin by using a mouse swimming model.

\section{Materials and Methods}

\subsection{Animal Experiments}

Three-week-old male Balb/c mice were purchased and fed a commercial non-purified chow diet (CRF-1, Charles River Laboratories Japan Inc.) for 2 weeks. The mice were kept in individual plastic cages at $23^{\circ} \mathrm{C} \pm 2{ }^{\circ} \mathrm{C}$ with a 12-hour light-dark cycle (light from 8 a.m. to 8 p.m.). During this period, the animals had 3 swimming training sessions, 15 min each. After the training, the 
animals were divided into 3 experimental groups $(n=6)$ : control group, rooibos group, and aspalathin group. Fast/slow swimmers distributed evenly among the groups. The diet fed to the control group was AIN-93G. The diets of the rooibos group or aspalathin groups comprised AIN-93G containing $0.2 \%$ of rooibos or $0.04 \%$ of aspalathin, respectively. The aspalathin content of the diets of rooibos group and aspalathin groups was the same. These contents were decided as minimum contents that have beneficial effects at our pilot study. Diet and drinking water were provided ad libitum.

After the distribution into the test groups, the animals were fed the experimental diets for 5 weeks. After 2 and 4 weeks, swimming tests were performed using a swimming instrument (Anitec, Japan). When the nose tip of each mouse was submerged in water for $7 \mathrm{~s}$, the test was marked as complete, and the swimming time was recorded. Water flow was maintained at $19 \mathrm{~cm} / \mathrm{s}$, and water temperature at $34^{\circ} \mathrm{C}-35^{\circ} \mathrm{C}$.

At the end of the experimental period, after the mice swam for $30 \mathrm{~min}$ (the maximum time that the animals can be made to swim), mice were sacrificed and blood and some organs such as the heart, liver, spleen, kidney, epididymal white adipose tissue, and skeletal muscle (gastrocnemius and soleus), were collected. The blood was centrifuged for $10 \mathrm{~min}$ at $1900 \mathrm{~g}$ to obtain plasma. Plasma, liver, and skeletal muscle were stored at $-30^{\circ} \mathrm{C}$ until analysis.

All animal studies were performed according to the approved animal research protocol of Showa Women's University.

\subsection{Blood Analysis}

The Blood glucose and lactic acid values were measured at the time of acrifice. Medisafe Chips (Terumo Corp., Tokyo, Japan) were used to measure blood glucose, and Lactate Pro test meter (Arkray Inc., Kyoto, Japan) was used to measure lactic acid. Manufacturers' protocols were followed for each analysis and all measurements were performed in duplicate.

Plasma free fatty acid levels were measured using the NEFA C-test kit (Wako Pure Chem., Osaka, Japan). Plasma activity of creatine kinase (CK) was measured using the CPK test kit (Wako Pure Chem., Osaka, Japan). The activities of aspartate aminotransferase (AST) and alanine aminotransferase (ALT) were measured using the transaminase C-test kit (Wako Pure Chem., Osaka, Japan).

\subsection{Glycogen Analysis}

Measurement of glycogen content in the liver was performed as described by Tashiro [7,8]. First, $50 \mathrm{mg}$ of liver was homogenized in $0.4 \mathrm{~mL}$ of $10 \%$ trichloroacetic acid and centrifuged at $10,000 \mathrm{~g}$ for $5 \mathrm{~min}$. Supernatant was recovered and 2 volumes of $95 \%$ ethanol was added, mixed thoroughly, and centrifuged at $1700 \mathrm{~g}$ for $5 \mathrm{~min}$. Glycogen was precipitated in a test tube, the supernatant was removed, and $0.5 \mathrm{~mL}$ of distilled water was added and mixed thoroughly. After mixing in $0.5 \mathrm{~mL}$ of $5 \%$ phenol, $2.5 \mathrm{~mL}$ of concentrated sulfuric acid was added, and the mixture was incubated at room temperature for 20 minutes. The absorbance at $490 \mathrm{~nm}$ was measured using a spectrophotometer and the data were used to calculate the glycogen concentration.

\subsection{Statistical Analysis}

The data have been represented as the means \pm SE. Statistical analysis was performed by analysis of variance using Tukey's multiple comparison. The differences were considered significant at $\mathrm{P}<0.05$.

\section{Results}

\subsection{Body and Organ Weights and Food Intake}

The body weight, food intake, and weight of some organs measured at the end of the experimental period are shown in Table 1. Mice in the 3 dietary groups demonstrated normal growth during the 5 weeks when they were fed the experimental diets. No significant differences were observed between the 3 groups in terms of body weight, food intake, and weight of the heart, liver, spleen, kidney, epididymal white adipose tissue, and skeletal muscle (gastrocnemius and soleus).

\subsection{Relative Swimming Time}

Relative swimming times at 0,2 and 4 weeks are shown in Figure 1. At week 2 and 4, the relative swimming times of the mice in the rooibos and aspalathin groups were longer than those of the control animals, although the difference was not significant.

Table 1. Body weight, food intake and weights of organs.

\begin{tabular}{cccc}
\hline & Control & Rooibos & Aspalathin \\
\hline Initial body weight (g) & $23.9 \pm 0.5$ & $23.3 \pm 0.6$ & $23.0 \pm 0.5$ \\
Final body weight (g) & $27.7 \pm 0.5$ & $28.0 \pm 0.6$ & $27.1 \pm 0.5$ \\
Food intake (g/day) & $4.5 \pm 0.7$ & $4.2 \pm 0.7$ & $4.0 \pm 0.6$ \\
Heart (mg) & $142 \pm 7$ & $155 \pm 5$ & $151 \pm 5$ \\
Liver (mg) & $1121 \pm 22$ & $1135 \pm 51$ & $1065 \pm 21$ \\
Spleen (mg) & $98 \pm 6$ & $125 \pm 14$ & $107 \pm 4$ \\
Kieney (mg) & $211 \pm 12$ & $209 \pm 17$ & $196 \pm 9$ \\
WAT (mg) & $299 \pm 23$ & $252 \pm 15$ & $254 \pm 33$ \\
Skeletal muscle (mg) & $59 \pm 5$ & $60 \pm 5$ & $50 \pm 3$ \\
\hline
\end{tabular}

Values are means \pm SE. Significant test was performed by analysis of variance using Tukey's multiple comparison. 


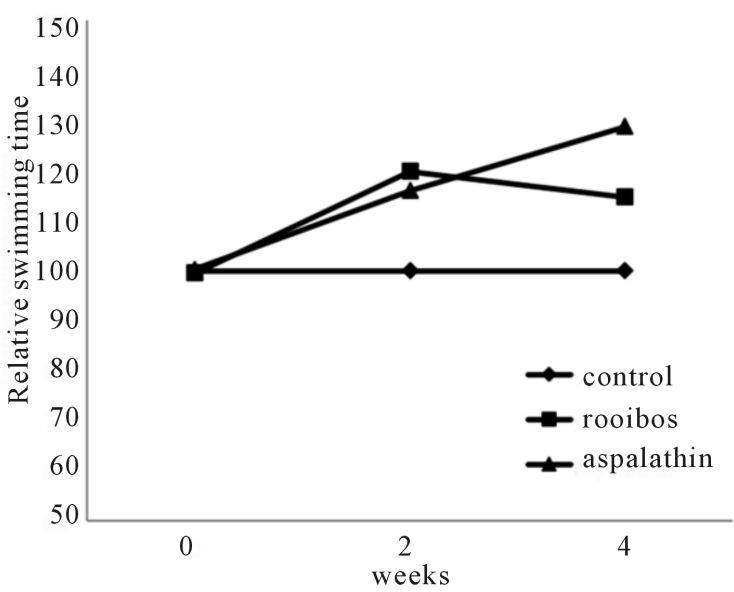

Figure 1. Relative swimming times at 0,2 and 4 weeks.

\subsection{Levels of Lactate and Glucose in Blood}

The levels of lactate (a) and glucose (b) in the blood of mice after swimming for 30 min are shown in Figure 2, respectively. Blood glucose level in the rooibos group was significantly lower than that in the other 2 groups. No significant difference in the blood lactate level was detected among the 3 dietary groups.

\subsection{Plasma Free Fatty Acid Levels}

The free fatty acid levels in the plasma of mice after swimming for 30 min are shown in Figure 3. No significant difference was detected among the 3 dietary groups.

\subsection{Plasma Creatine Kinase Activity}

The CK activity in the plasma of mice after swimming for $30 \mathrm{~min}$ is shown in Figure 4. No significant difference was detected among the 3 dietary groups.

\subsection{Plasma Aspartate Aminotransferase and Alanine Aminotransferase Activities}

AST (a) and ALT (b) activities in plasma of mice after swimming for 30 min are shown in Figure 5, respectively. No significant differences in the activities of these enzymes were detected among the 3 dietary groups.

\subsection{Glycogen Contents in the Liver and Skeletal Muscles}

Glycogen contents in the liver (a) and skeletal muscles (b) of mice after 30 min swimming are shown in Figure 6, respectively. Glycogen contents in the livers of the rooibos and aspalathin groups were significantly higher than that in the livers of the control group. Similarly, glycogen content in the skeletal muscles of the rooibos and aspalathin groups was significantly higher than that in the muscles of the control group.
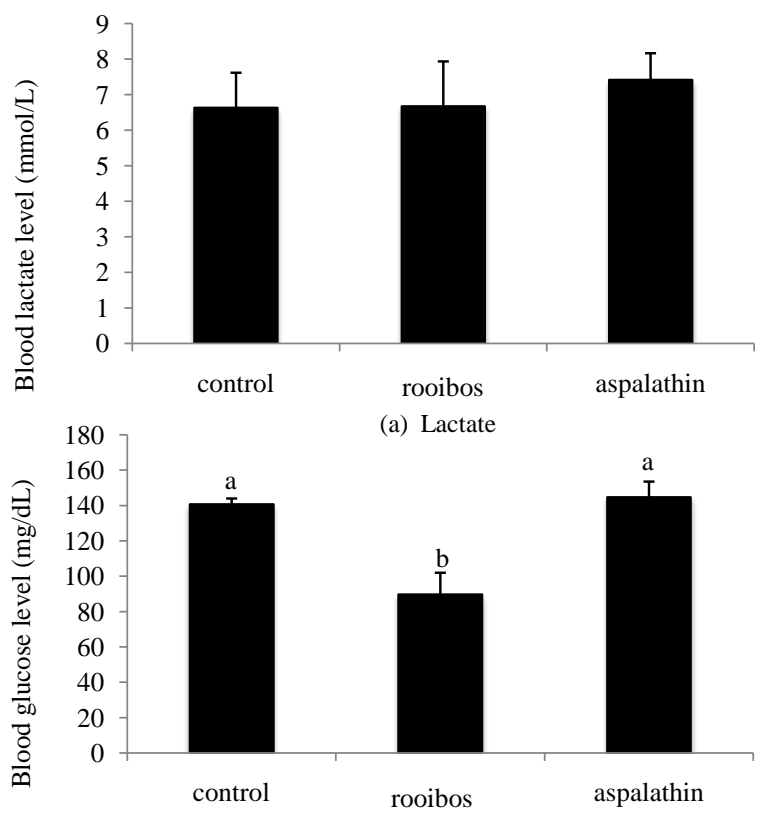

(b) Glucose

Figure 2. Blood levels of lactate (a) and glucose (b) of mice after swimming for $30 \mathrm{~min}$. Different letters above the bars indicate significant differences among groups $(P<0.05)$. Values are means $\pm \mathrm{SE}$.

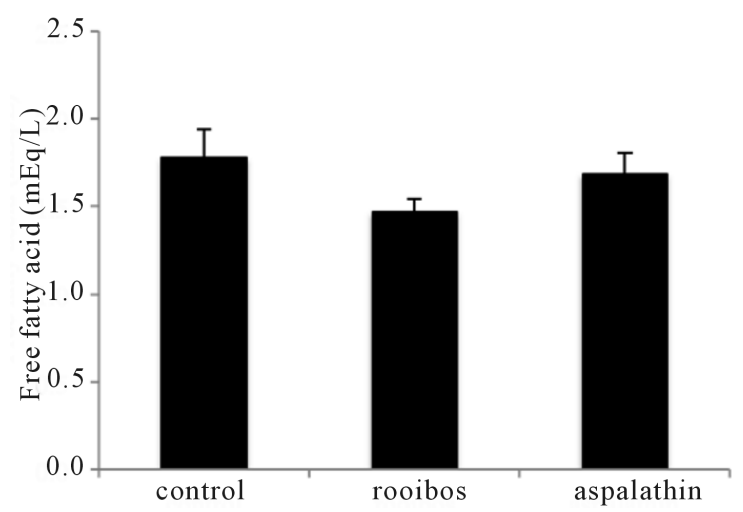

Figure 3. Plasma free fatty acid levels of mice after swimming for $30 \mathrm{~min}$. Values are means $\pm \mathrm{SE}$.

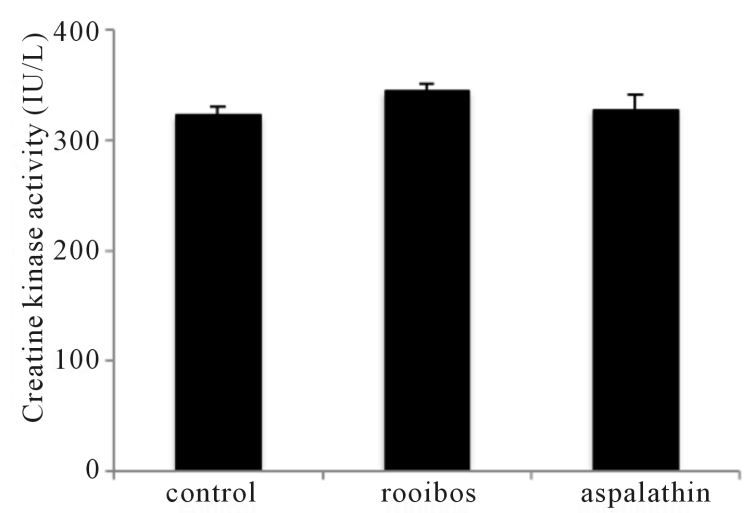

Figure 4. Plasma CK activities of mice after swimming for $30 \mathrm{~min}$. Values are means $\pm \mathrm{SE}$. 


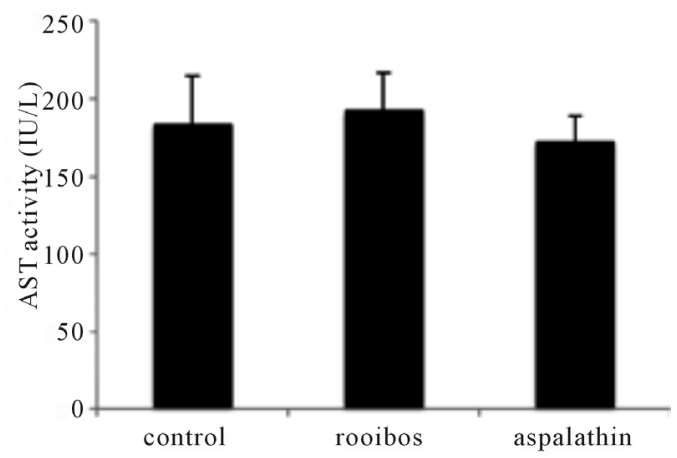

(a) AST

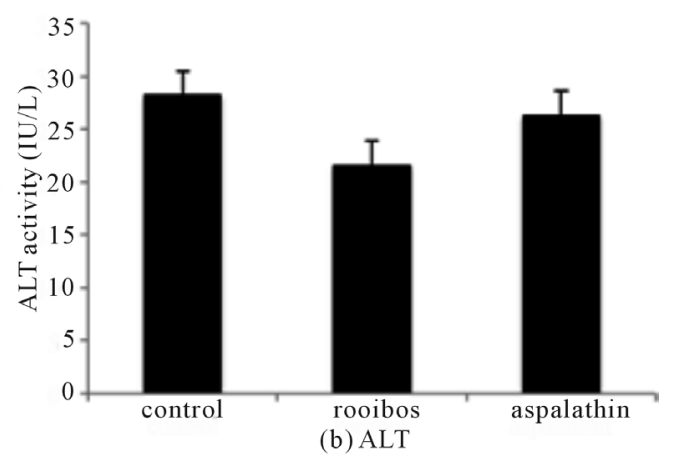

Figure 5. Plasma activities of AST (a) and ALT (b) of mice after swimming for $30 \mathrm{~min}$. Values are means $\pm \mathrm{SE}$.
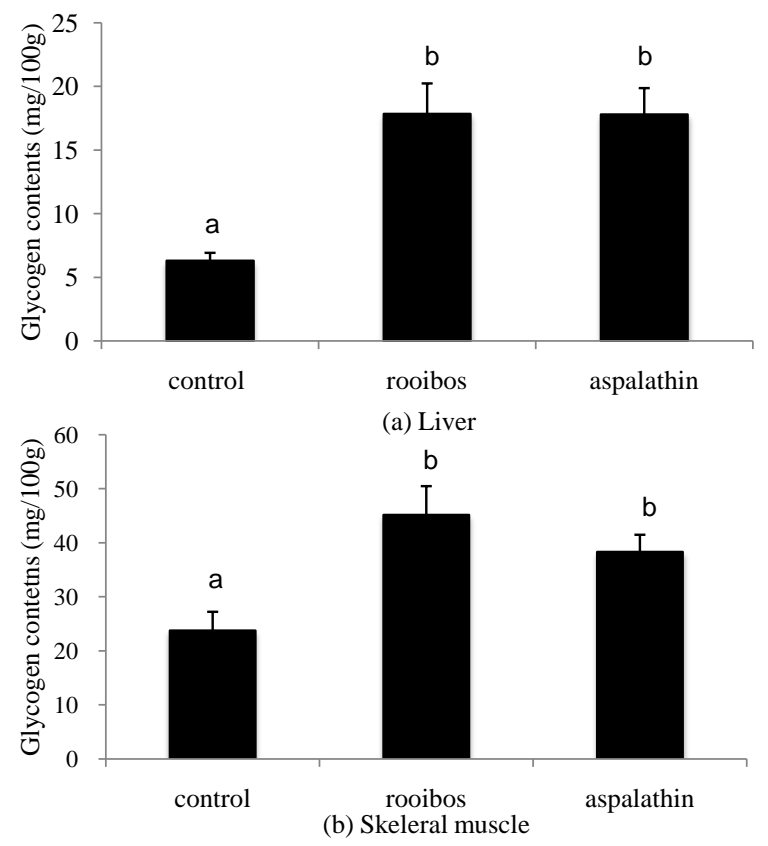

Figure 6. Glycogen contents in the liver (a) and skeletal muscles (b) of mice after 30 min swimming. Different letters above the bars indicate significant differences among groups $(\mathrm{P}<\mathbf{0 . 0 5})$. Values are means $\pm \mathrm{SE}$.

\section{Discussion}

The swimming times of the animals in the rooibos and aspalathin groups after 2 and 4 weeks on diets were longer than those in the control group. Although the difference was not significant, the rooibos and aspalathin might have the potential to increase physical endurance and decrease fatigue. If the beneficial effects of rooibos and aspalathin are confirmed, their activities need to be elucidated.

The plasma tests conducted in mice after 30 min of swimming revealed that the rooibos and aspalathinenriched diets did not decrease muscle damages, because plasma levels of CK, AST, and ALT levels were not significantly different among the 3 dietary groups. Free fatty acid content in plasma was also not significantly different among the 3 dietary groups, suggesting that dietary rooibos and aspalathin did not increase the utilization of free fatty acids induced by the activation of hormonesensitive lipase.

On the other hand, the glycogen contents in the liver and skeletal muscles of the animals in the rooibos and aspalathin groups were significantly higher than those in the control group. These results suggest that dietary rooibos possibly accelerates accumulation of glycogen in the liver and skeletal muscle through the activity of aspalathin, which also prolonged the swimming time of mice. The increased glycogen accumulation in the liver and skeletal muscles has been suggested to improve physical endurance $[9,10]$. Gina et al. also showed that rats have high contents in the soleus muscle, which indicates strong resistance from swim exhaustion [11]. Aspalathin present in rooibos has also been shown to increase glucose intake via the up-regulation of GLUT4 translocation to the cell membrane [4-6], which may stimulate glycogen accumulation.

The other mechanism underlying prolongation of swimming time can be induced by the antioxidative activity of dietary components [12,13]. Increased intake of 3,4-dihydroxycinnamic acid known as one of the strongest antioxidants, decreased blood lactate levels and increased physical endurance in rats [12]. Dietary ferulic acid, another antioxidant, also increased physical endurance and decreased fatigue in swimming mice [13]. Apart from aspalathin, rooibos contains other dihydrochalcones such as nothofagin, which have antioxidative activity [14]. Therefore, it is likely that the beneficial effects of rooibos can be attributed to aspalathin and other dihydrochalcones.

These results suggest that dietary green rooibos has the potential to improve physical endurance due to the antioxidative properties of its components such as aspalathin.

\section{REFERENCES}

[1] L. Bramati, F. Aquilano and P. Pietta, "Unfermented Rooibos Tea: Quantitative Characterization of Flavonoids by HPLC-UV and Determination of the Total Antioxidant Activity," Journal of Agricultural and Food Chemistry, 
Vol. 51, No. 25, 2003, pp. 7472-7474. http://dx.doi.org/10.1021/jf0347721

[2] D. Villaño, M. Pecorari, M. F. Testa, A. Raguzzini, A. Stalmach, A. Crozier, C. Tubili and M. Serafini, "Unfermented and Fermented Rooibos Teas (Aspalathus linearis) Increase Plasma Total Antioxidant Capacity in Healthy Humans," Food Chemistry, Vol. 123, No. 3, 2010, pp. 679-683. http://dx.doi.org/10.1016/j.foodchem.2010.05.032

[3] G. P. Novelli, G. Bracciotti and S. Falsini, "Spin-Trappers and Vitamin E Prolong Endurance to Muscle Fatigue In mice,” Free Radical Biology and Medicine, Vol. 8, No. 1, 1990, pp. 9-13. http://dx.doi.org/10.1016/0891-5849(90)90138-9

[4] A. Kawano, H. Nakamura, S. Hata, M. Minakawa, Y. Miura and K. Yagasaki, "Hypoglycemic Effect of Aspalathin, a Rooibos Tea Component from Aspalathus linearis, in Type 2 Diabetic Model db/db Mice,” Phytomedicine, Vol. 15, No. 5, 2009, pp. 437-443. http://dx.doi.org/10.1016/j.phymed.2008.11.009

[5] B. M. S. Giambastiani, "Evoluzione Idrologica ed Idrogeologica Della Pineta di San Vitale (Ravenna),” Ph.D. Thesis, Bologna University, Bologna, 2007.

[6] M. J. Son, M. Minakawa, Y. Miura and K. Yagasaki, "Aspalathin Improves Hyperglycemia and Glucose Intolerance in Obese Diabetic ob/ob Mice," European Journal of Nutrition, Vol. 52, No. 6, 2013, pp. 1607-1619. http://dx.doi.org/10.1007/s00394-012-0466-6

[7] M. Tashiro, “An Introduction to Practical Biochemistry,” Kagaku-Dojin Publishing Company Inc., Kyoto, 2006.

[8] O. Wright and W. Wright, "Flying-Machine," US Patent No. 821393, 1906.

[9] A. Shinohara, M. Suzuki, J. Takakura and A. Yamane, "Effect of the Classic 1-Week Glycogen-Loading Regimen on Fat-Loading in Rats and Humans," Journal of Nutri- tional Science and Vitaminology, Vol. 56, No. 5, 2010, pp. 299-304. http://dx.doi.org/10.3177/jnsv.56.299

[10] J. A. De Araujo, G. Falavigna, M. M. Rogero, I. S. O. Pires, R. G. Pedrosa, I. A. Castro, J. Donato and J. Tirapegui, "Effect of Chronic Supplementation with BranchedChain Amino Acids on the Performance and Hepatic and Muscle Glycogen Content in Trained Rats,” Life Sciences, Vol. 79, No. 14, 2006, pp. 1343-1348. http://dx.doi.org/10.1016/j.lfs.2006.03.045

[11] G. Falavigna, J. Alves de Araújo Jr, M. M. Rogero, I. S. Pires, R. G. Pedrosa, E. Martins Jr, I. Alves de Castro and J. Tirapegui, "Effects of Diets Supplemented with BranchedChain Amino Acids on the Performance and Fatigue Mechanisms of Rats Submitted to Prolonged Physical Exercise,” Nutrients, Vol. 4, No. 11, 2012, pp. 1767-1780. http://dx.doi.org/10.3390/nu4111767

[12] R. D. Novaes, R. V. Goncalves, I. R. S. C. Maldonado, M. D. C. G. Peluzio and A. J. Natali, "3,4-Dihydroxycinnamic Acid Attenuates the Fatigue and Improves Exercise Tolerance in Rats," Bioscience, Biotechnology, and Biochemistry, Vol. 76, No. 5, 2012, pp. 1025-1027. http://dx.doi.org/10.1271/bbb.111006

[13] Y. You, J. Park, W. Jun, H. G. Yoon, Y. H. Lee, K. Hwang, J. Lee, K. Kim, K. W. Lee and S. Shin, "Stimulatory Effects of Ferulic Acid on Endurance Exercise Capacity in Mice,” Bioscience, Biotechnology, and Biochemistry, Vol. 73, No. 6, 2009, pp. 1392-1397. http://dx.doi.org/10.1271/bbb.111006

[14] V. D. M. K. Debora, J. Elizabeth, M. Marena, C. A. G. Wentzel, J. Elizabeth, D. B. Dalene, J. M. Christiaan and C. A. G. Wentzel, "In Vitro Hepatic Biotransformation of Asparathin and Nothofagin, Dihydrochalcones of Rooibos (Aspalathus linearis), and Assessment of Metabolite Antioxidant Activity,” Journal of Agricultural and Food Chemistry, Vol. 58, No. 4, 2010, pp. 2214-2220. http://dx.doi.org/10.1021/jf903917a

\begin{abstract}
Abbreviations
GLUT4, glucose transporter type 4; AMPK, adenosine monophosphate-activated protein kinase; NEFA, non-esterified fatty acid; CK, creatine kinase; AST, asparatate aminotransferase; $\mathrm{ALT}$, alanine aminotransferase.
\end{abstract}

